\title{
Angle of Arrival Estimation Using Cholesky Decomposition
}

\author{
Saleh O. Al-Jazzar \\ Electrical Engineering Department, University of Ha’il, Ha’il 81451, Saudi Arabia \\ Correspondence should be addressed to Saleh O. Al-Jazzar, s.jazzar@uoh.edu.sa
}

Received 23 November 2011; Revised 22 February 2012; Accepted 5 March 2012

Academic Editor: Stefano Selleri

Copyright (C) 2012 Saleh O. Al-Jazzar. This is an open access article distributed under the Creative Commons Attribution License, which permits unrestricted use, distribution, and reproduction in any medium, provided the original work is properly cited.

\begin{abstract}
An angle of arrival (AOA) estimator is presented. Many applications require accurate AOA estimates such as wireless positioning and signal enhancement using space-processing techniques. The proposed AOA estimator depends on the Cholesky decomposition of the received signal autocorrelation matrix. The resultant decomposed matrices are used to modify the crosscorrelation matrix of the received signals at the antenna array doublets. The proposed method is named the Cholesky-decomposition-based-AOA (CDBA) estimator. In comparison with the TLS-ESPRIT algorithm which utilizes the eigenvalue decomposition (EVD) of the received signal autocorrelation and crosscorrelation matrices, the CDBA method has better performance than the TLS-ESPRIT algorithm especially in low signal-to-noise-ratio (SNR) cases. Simulations for the proposed CDBA method are shown to assess its performance.
\end{abstract}

\section{Introduction}

Accurate estimation of the received signal angle of arrival (AOA) can be very beneficial for signal reception enhancement $[1,2]$ and wireless positioning $[3,4]$. If the AOA estimates were not estimated accurately, then wireless devices would not be located accurately or the received signal bit error rate would be high. Thus, AOA estimators are developed and modified in such cases to increase their accuracy.

Many AOA estimators were proposed in the literature [5-9]. The authors in [5] present an iterative method to estimate the AOA. In [6], the authors propose estimating the AOA using the frequency domain of the received signals which requires taking their Fourier transform. The authors in [7] introduce an iterative AOA estimator which depends on the generalized expectation maximization algorithm. In [8], the authors present an AOA estimator for circular arrays. Another very popular AOA estimator is the TLS-ESPRIT algorithm proposed in [9] which utilizes subspace techniques to estimate the AOA.

In this paper, an AOA estimator is proposed which enhances the AOA estimation. The proposed method is named the Cholesky-decomposition-based-AOA (CDBA) estimator. The CDBA method utilizes the received signal at the two sides of the antenna array doublets. An autocorrelation matrix of the received signal at one side of the antenna array doublets is calculated. Then, the received signal autocorrelation matrix is decomposed using the Cholesky decomposition. A crosscorrelation matrix between the received signals at both sides of the antenna doublets is calculated. The decomposed matrices of the autocorrelation matrix together with the crosscorrelation matrix are used to form a new matrix from which the AOAs are estimated.

The CDBA method does not require taking the Fourier transform of the received signals nor it requires any iterative or searching procedure to estimate the AOA. Also, in comparison with the well-known TLS-ESPRIT algorithm of [9], the accuracy of the CDBA method is higher than that of the TLS-ESPRIT method especially in low signal-to-noise-ratio (SNR) cases.

The paper is organized as follows: Section 2 introduces the system model that forms the foundation for the proposed estimator. The proposed CDBA estimator is presented in Section 3. Section 4 presents the simulated performance of the CDBA estimator. Finally, conclusions are shown in Section 5 .

\section{System Model}

This section presents the narrowband received signal model that will be utilized for the AOA estimation. The antenna 
array is formed from $M$ uniform antenna doublets (i.e., $2 M$ total antenna elements). Each antenna doublet is formed of two antenna elements spaced by a distance $d_{d}$. We assume a $K$ BPSK sources signals, $\left\{\tilde{s}_{k}(t)\right\}_{k=1}^{K}$, impinging upon the antenna array. The signal $\widetilde{s}_{k}(t)$ is represented as $\widetilde{s}_{k}(t)=$ $s_{k}(t) \cos \left(2 \pi f_{c} t\right)=\mathfrak{R}\left\{s_{k}(t) \exp \left(j 2 \pi f_{c} t\right)\right\}$, where $f_{c}$ is the carrier frequency $s_{k}(t)=\sum_{i} \alpha_{k}(i) g(t-i T), i$ is an integer which represents the time index and $s_{k}(t)$ is the complex low-pass equivalent of $\tilde{s}_{k}(t), T$ is the symbol period, $\alpha_{k}(i)=$ $A_{k} \beta_{k}(i)$, where $\beta_{k}(i)$ is discrete $\beta_{k}(i) \in\{-1,+1\}$ which represents the symbol parity and $A_{k}$ is a positive constant which represents the amplitude of $s_{k}(t)$, and $g(t)$ is the (raised cosine) pulse-shaping function with

$$
g(n T)= \begin{cases}1 & \text { if } n=0 \\ 0 & \text { otherwise }\end{cases}
$$

where $n$ is an integer so that $s_{k}(n T)=\alpha_{k}(n)$.

The AOA is taken between the antenna array axis, and the source signal arrival direction and will be given the notation $\left(\theta_{k}\right)$. In this paper, the superscript notation \{\} is used to denote the estimated value of a variable (for example $\hat{h}$ is the estimated value of $h$ ). So, to start developing the received signal model, let us first consider the sampled received signal at the antenna elements (after the matched filter stage), from $m=1$ to $m=M$, that are located in the first side of the antenna doublets, and let us call it $\mathbf{r}_{1}(i)$, and is represented as

$$
\mathbf{r}_{1}(i)=\sum_{k=1}^{K} \alpha_{k}(i) \mathbf{a}\left(\theta_{k}\right)+\mathbf{n}_{1}(i)
$$

where

$$
\begin{gathered}
\mathbf{a}\left(\theta_{k}\right)=\left[\begin{array}{lll}
a_{1}\left(\theta_{k}\right) & \cdots & a_{M}\left(\theta_{k}\right)
\end{array}\right]^{T}, \\
a_{m}\left(\theta_{k}\right)=\exp \left\{j \frac{2 \pi}{\lambda} d \times(m-1) \cos \left(\theta_{k}\right)\right\}
\end{gathered}
$$

where $\lambda$ is the signal wavelength, and $d$ is the distance between consecutive antenna elements. In addition, $\mathbf{n}_{1}(i)$ is the noise vector added to the received signal at the antenna elements (from $m=1 \rightarrow M$ ) located along the first side of the antenna doublets, which is additive white Gaussian noise (AWGN) and has a covariance matrix of $\sigma^{2} \mathbf{I}_{M \times M}$, where $\mathbf{I}_{M \times M}$ is the $M \times M$ identity matrix. Finally, $(\cdot)^{T}$ represents the transpose operation.

The $(M \times 1)$ received signal vector at the first side of the antenna doublets set can be written in matrix form as

$$
\mathbf{r}_{1}(i)=\mathbf{A}(\boldsymbol{\theta}) \boldsymbol{\alpha}(i)+\mathbf{n}_{1}(i),
$$

where

$$
\begin{aligned}
\mathbf{A}(\boldsymbol{\theta}) & =\left[\begin{array}{lll}
\mathbf{a}\left(\theta_{1}\right) & \cdots & \mathbf{a}\left(\theta_{K}\right)
\end{array}\right], \\
\boldsymbol{\theta} & =\left[\begin{array}{lll}
\theta_{1} & \cdots & \theta_{K}
\end{array}\right]^{T}, \\
\boldsymbol{\alpha}(i) & =\left[\begin{array}{lll}
\alpha_{1}(i) & \cdots & \alpha_{K}(i)
\end{array}\right]^{T} .
\end{aligned}
$$

From now on the term (i) will be dropped from all terms for simplicity.

The received signal vector at the second side of the antenna doublets set will be given the notation $\mathbf{r}_{2}$. Thus,

$$
\mathbf{r}_{2}=\mathbf{A}(\boldsymbol{\theta}) \mathbf{Z} \boldsymbol{\alpha}+\mathbf{n}_{2},
$$

where $\mathbf{n}_{2}$ is an AWGN vector at the second side of the antenna array doublets, and

$$
\mathbf{Z}=\operatorname{diag}\left(\left[\begin{array}{lllll}
z_{1} & \cdots & z_{k} & \cdots & z_{K}
\end{array}\right]\right)
$$

with

$$
z_{k}=\exp \left\{j \frac{2 \pi}{\lambda} d_{d} \times \cos \left(\theta_{k}\right)\right\}
$$

Next section will describe the proposed CDBA estimator.

\section{Proposed Cholesky-Decomposition-Based AOA (CDBA) Estimator}

To implement the CDBA method, the crosscorrelation matrix $\left(\mathbf{R}_{21}\right)$ between $\mathbf{r}_{2}$ and $\mathbf{r}_{1}$ is formulated as follows:

$$
\mathbf{R}_{21}=E\left[\mathbf{r}_{2} \mathbf{r}_{1}^{H}\right]=\mathbf{A}(\boldsymbol{\theta}) \mathbf{P}_{s} \mathbf{Z A}(\boldsymbol{\theta})^{H}
$$

where

$$
\mathbf{P}_{s}:=E\left[\boldsymbol{\alpha} \boldsymbol{\alpha}^{H}\right]=\operatorname{diag}\left(\left[p_{1} \cdots p_{k} \cdots p_{K}\right]\right),
$$

where $p_{k}$ is the power of the $k$ th source.

Another matrix considered by the CDBA algorithm is the autocorrelation matrix of $\mathbf{r}_{1}$, as follows:

$$
\widetilde{\mathbf{R}}_{11}=E\left[\mathbf{r}_{1} \mathbf{r}_{1}^{H}\right]=\mathbf{A}(\boldsymbol{\theta}) \mathbf{P}_{s} \mathbf{A}(\boldsymbol{\theta})^{H}+\sigma^{2} \mathbf{I}_{M \times M} .
$$

Take the noise free version of $\widetilde{\mathbf{R}}_{11}$, and let us call it $\mathbf{R}_{11}$, where $\mathbf{R}_{11}=\widetilde{\mathbf{R}}_{11}-\sigma^{2} \mathbf{I}_{M \times M}$, that is,

$$
\mathbf{R}_{11}=\mathbf{A}(\boldsymbol{\theta}) \mathbf{P}_{s} \mathbf{A}(\boldsymbol{\theta})^{H} .
$$

Then, the eigenvalue decomposition (EVD) of $\mathbf{R}_{11}$ is

$$
\mathbf{R}_{11}=\mathbf{V} \boldsymbol{\Gamma} \mathbf{V}^{H},
$$

where $\mathbf{V}$ is the eigenvector matrix of $\mathbf{R}_{11}$, and $\boldsymbol{\Gamma}$ is an $M \times M$ diagonal matrix with its diagonal that contains the eigenvalues of $\mathbf{R}_{11}$, that is,

$$
\boldsymbol{\Gamma}=\operatorname{diag}\left(\left[\begin{array}{llllll}
\gamma_{1} & \cdots & \gamma_{K} & 0 & \cdots & 0
\end{array}\right]\right),
$$

where the $K$ nonzero values of the diagonal elements of $\boldsymbol{\Gamma}$ correspond to the $K$ sources which will be called $\gamma_{1 \rightarrow K} \equiv$ $\gamma_{1} \rightarrow \gamma_{K}$. Each element of $\gamma_{1 \rightarrow K}$ corresponds to one of the $K$ sources.

Comparing (12) with (13), then we have

$$
\mathbf{A}(\boldsymbol{\theta}) \mathbf{P}_{s} \mathbf{A}(\boldsymbol{\theta})^{H}=\mathbf{V} \boldsymbol{\Gamma} \mathbf{V}^{H} .
$$


Comparing both sides of (15), then we have

$$
\mathbf{A}(\boldsymbol{\theta})=\mathbf{V T},
$$

where $\mathbf{T}$ is the appropriate matrix to change the basis.

Substituting (16) into (15), then we have

$$
\Gamma=\mathbf{T P}_{s} \mathbf{T}^{H} .
$$

The matrix $\boldsymbol{\Gamma}$ is an $M \times M$ matrix, and $\mathbf{P}_{s}$ is a $K \times K$ diagonal matrix. Also, the nonzero diagonal elements of $\Gamma$ are only the first $K$ diagonal elements. So, (17) can be written as

$$
\left[\begin{array}{cccccc}
\gamma_{1} & \cdots & 0 & 0 & \cdots & 0 \\
\vdots & \ddots & \vdots & \vdots & \ddots & \vdots \\
0 & \cdots & \gamma_{K} & 0 & \cdots & 0 \\
0 & \cdots & 0 & 0 & \cdots & 0 \\
\vdots & \ddots & \vdots & \vdots & \ddots & \vdots \\
0 & \cdots & 0 & 0 & \cdots & 0
\end{array}\right]_{M \times M}=\mathbf{T}\left[\begin{array}{ccc}
p_{1} & \cdots & 0 \\
\vdots & \ddots & \vdots \\
0 & \cdots & p_{K}
\end{array}\right]_{K \times K} \mathbf{T}^{H} .
$$

Looking at (18), it can be deduced that the matrix $\mathbf{T}$ should be $M \times K$. Also, the lower $(M-K) \times K$ part of $\mathrm{T}$ should be all zero elements, that is,

$$
\mathbf{T}=\left[\begin{array}{ccc}
t_{1,1} & \cdots & t_{1, K} \\
\vdots & & \vdots \\
t_{K, 1} & \cdots & t_{K, K} \\
0 & \cdots & 0 \\
\vdots & & \vdots \\
0 & \cdots & 0
\end{array}\right]_{M \times K}=\left[\begin{array}{c}
\mathbf{\Omega} \\
\mathbf{0}
\end{array}\right]
$$

where

$$
\boldsymbol{\Omega}=\left[\begin{array}{ccc}
t_{1,1} & \cdots & t_{1, K} \\
\vdots & & \vdots \\
t_{K, 1} & \cdots & t_{K, K}
\end{array}\right]_{K \times K} .
$$

But, the left hand side of (18) is a diagonal matrix, and the matrix $\mathbf{P}_{s}$ is diagonal as well. So, from (18) it can deduced that the matrix $\boldsymbol{\Omega}$ is diagonal too, that is,

$$
\boldsymbol{\Omega}=\left[\begin{array}{ccc}
t_{1,1} & \cdots & 0 \\
\vdots & & \vdots \\
0 & \cdots & t_{K, K}
\end{array}\right]_{K \times K} .
$$

Thus, from (18), (19), and (21), each of the nonzero diagonal elements of $\Gamma$ is given by

$$
\gamma_{k}=p_{k}\left|t_{k, k}\right|^{2}
$$

which has a positive real value.

Consequently, the EVD of $\widetilde{\mathbf{R}}_{11}$ would be given by

$$
\widetilde{\mathbf{R}}_{11}=\mathbf{V} \widetilde{\Gamma} \mathbf{V}^{H},
$$

where $\tilde{\boldsymbol{\Gamma}}$ is an $M \times M$ diagonal matrix and is given by $\widetilde{\boldsymbol{\Gamma}}=$ $\boldsymbol{\Gamma}+\sigma^{2} \mathbf{I}_{M \times M}$, that is,

$$
\widetilde{\boldsymbol{\Gamma}}=\operatorname{diag}\left(\left[\gamma_{1}+\sigma^{2} \ldots \gamma_{\mathrm{K}}+\sigma^{2} \sigma^{2} \ldots \sigma^{2}\right]\right) \text {. }
$$

Substituting (16) into (23), then we have

$$
\widetilde{\mathbf{R}}_{11}=\mathbf{A}(\boldsymbol{\theta}) \mathbf{T}^{+} \tilde{\boldsymbol{\Gamma}} \mathbf{T}^{+H} \mathbf{A}(\boldsymbol{\theta})^{H},
$$

where the notation $(\cdot)^{+}$is the pseudoinverse for a given matrix.

Now, taking the Cholesky decomposition of $\widetilde{\mathbf{R}}_{11}$, then we have

$$
\widetilde{\mathbf{R}}_{11}=\widetilde{\mathbf{L}} \widetilde{\mathbf{L}}^{H}
$$

where $\tilde{\mathbf{L}}$ is a lower triangular matrix that results from the Cholesky decomposition.

Comparing (26) with (25), it can be deduced that

$$
\widetilde{\mathbf{L}}=\mathbf{A}(\boldsymbol{\theta}) \mathbf{T}^{+} \widetilde{\boldsymbol{\Gamma}}^{1 / 2} \mathbf{F}
$$

where $\mathbf{F}$ is an orthogonal rotating matrix.

A new matrix $(\Psi)$ is formed by multiplying the left and right hand sides of $\mathbf{R}_{21}$ by $\widetilde{\mathbf{L}}^{-1}$ and $\tilde{\mathbf{L}}^{-H}$, respectively, as follows:

$$
\boldsymbol{\Psi}=\widetilde{\mathbf{L}}^{-1} \mathbf{R}_{21} \widetilde{\mathbf{L}}^{-H}
$$

But, $\mathbf{R}_{21}=\mathbf{A}(\boldsymbol{\theta}) \mathbf{P}_{s} \mathbf{Z A}(\boldsymbol{\theta})^{H}$ as shown in (9) and $\widetilde{\mathbf{L}}=\mathbf{A}(\boldsymbol{\theta})$ $\mathbf{T}^{+} \widetilde{\boldsymbol{\Gamma}}^{1 / 2} \mathbf{F}$ as shown in (27). Thus,

$$
\boldsymbol{\Psi}=\mathbf{F}^{-1} \widetilde{\boldsymbol{\Gamma}}^{-1 / 2} \mathbf{T P}_{s} \mathbf{Z} \mathbf{T}^{H} \widetilde{\boldsymbol{\Gamma}}^{-H / 2} \mathbf{F}^{-H} .
$$

Now, let

$$
\boldsymbol{\Phi}=\widetilde{\boldsymbol{\Gamma}}^{-1 / 2} \mathbf{T} \mathbf{P}_{s} \mathbf{Z} \mathbf{T}^{H} \widetilde{\boldsymbol{\Gamma}}^{-H / 2}
$$

Also, let $\mathbf{G}=\mathbf{F}^{-1}$. Then, (29) can be written as

$$
\Psi=\mathbf{G} \Phi \mathbf{G}^{H} .
$$

Looking at (31), if it is proved that the matrix $\Phi$ is a diagonal matrix, then (31) will be the EVD of $\boldsymbol{\Psi}$, where $\mathbf{G}$ and $\boldsymbol{\Phi}$ are the corresponding eigenvectors and eigenvalues matrices, respectively.

To prove that $\Phi$ is a diagonal matrix, consider the matrix $\widetilde{\Gamma}^{-1 / 2}$ which is a diagonal matrix of size $M \times M$ and the matrix $\mathrm{T}$ which is an $M \times K$ matrix where its upper $K \times K$ part is diagonal, and its lower $(M-K) \times K$ part contains zero elements, then

$$
\widetilde{\boldsymbol{\Gamma}}^{-1 / 2} \mathbf{T}=\mathbf{T} \widetilde{\Gamma}_{s}^{-1 / 2}
$$

where $\tilde{\boldsymbol{\Gamma}}_{s}$ is a diagonal matrix of size $K \times K$ where its diagonal elements are the same as the first $K$ diagonal elements of $\widetilde{\boldsymbol{\Gamma}}$, that is,

$$
\widetilde{\boldsymbol{\Gamma}}_{s}=\operatorname{diag}\left(\left[\gamma_{1}+\sigma^{2} \cdots \gamma_{K}+\sigma^{2}\right]\right) .
$$

Thus, (30) can be written as

$$
\boldsymbol{\Phi}=\mathbf{T} \widetilde{\Gamma}_{s}^{-1 / 2} \mathbf{P}_{s} \mathbf{Z} \widetilde{\boldsymbol{\Gamma}}_{s}^{-H / 2} \mathbf{T}^{H}
$$


Since $\widetilde{\boldsymbol{\Gamma}}_{s}^{-1 / 2}, \mathbf{P}_{s}$ and $\mathbf{Z}$ are diagonal matrices, then we have

$$
\Phi=\mathbf{T}\left|\widetilde{\boldsymbol{\Gamma}}_{s}\right|^{-1} \mathbf{P}_{s} \mathbf{Z} \mathbf{T}^{H}
$$

But, the matrix $\widetilde{\boldsymbol{\Gamma}}_{s}$ is diagonal matrix where its elements are positive real. So, $\left|\widetilde{\boldsymbol{\Gamma}}_{s}\right|=\widetilde{\boldsymbol{\Gamma}}_{s}$, and

$$
\Phi=\mathbf{T} \tilde{\Gamma}_{s}^{-1} \mathbf{P}_{s} \mathbf{Z} \mathbf{T}^{H} .
$$

Substituting for $\mathbf{T}, \widetilde{\boldsymbol{\Gamma}}_{s}, \mathbf{P}_{s}$, and $\mathbf{Z}$ in (36), then we have

$$
\boldsymbol{\Phi}=\operatorname{diag}\left(\left[\begin{array}{llllll}
\phi_{1} & \cdots & \phi_{K} & 0 & \cdots & 0
\end{array}\right]\right)
$$

where

$$
\phi_{k}=\frac{p_{k}\left|t_{k, k}\right|^{2} z_{k}}{\gamma_{k}+\sigma^{2}} .
$$

But, $\gamma_{k}$ is defined in (22) as $\gamma_{k}=p_{k}\left|t_{k, k}\right|^{2}$. Thus, (38) can be written as

$$
\phi_{k}=\frac{\gamma_{k} z_{k}}{\gamma_{k}+\sigma^{2}} .
$$

Thus, it is clear that $\Phi$ is a diagonal matrix and it is proved now that (31) is the EVD of $\Psi$.

Defining the term

$$
y_{k}=\frac{\gamma_{k}}{\gamma_{k}+\sigma^{2}}
$$

then (39) can be written as

$$
\phi_{k}=y_{k} z_{k}
$$

Now, because $y_{k}$ has a positive real value, then

$$
\angle \phi_{k}=\angle z_{k}
$$

From the definition of $z_{k}$, it is clear that the AOAs are implicated in $\angle z_{k}$. Also, from (42) the phase of $z_{k}$ equals the phase of $\phi_{k}$, and $\phi_{k}$ for $k=1 \rightarrow K$ are the diagonal elements of $\boldsymbol{\Gamma}$. Thus, to find $\theta_{k}$, consider the $k$ th diagonal element of $\boldsymbol{\Phi}$ as follows:

$$
\hat{\theta}_{k}=\cos ^{-1}\left(\frac{\lambda \angle\left(\hat{\phi}_{k}\right)}{2 \pi d_{d}}\right),
$$

where $\hat{\phi}_{k}$ is the $k$ th diagonal element of the estimated $\hat{\boldsymbol{\Phi}}$.

Thus, the required AOAs are estimated.

\subsection{Summary of the CDBA Method.}

(1) First step: calculate $\mathbf{R}_{21}$ from (9).

(2) Second step: calculate $\widetilde{\mathbf{R}}_{11}$ from (11).

(3) Third step: calculate $\tilde{\mathbf{L}}$ by taking the Cholesky decomposition of $\widetilde{\mathbf{R}}_{11}$.

(4) Fourth step: calculate the $\boldsymbol{\Psi}$ matrix by $\boldsymbol{\Psi}=$ $\widetilde{\mathbf{L}}^{-1} \mathbf{R}_{21} \tilde{\mathbf{L}}^{-H}$.

(5) Fifth step: calculate the eigenvalues of $\Psi$ matrix.

(6) Sixth step: calculate the AOAs from the $K$ largest eigenvalues of $\Psi$ matrix as shown in (43).
3.2. Analysis for the Effect of Noise Variance. The performance of the AOA estimators in high noise power (low SNR) cases is of interest. A measure of the noise power is the noise variance. A study of the noise variance effect on the performance of the CDBA algorithm is derived and compared with that of the TLS-ESPRIT.

As shown in (43), the AOAs are estimated from the eigenvalues $\left(\phi_{k}\right)$. Thus, to start let us consider the effect of noise variance (power) on $\phi_{k}$.

Consider the magnitude of $\phi_{k}$ as follows:

$$
\left|\phi_{k}\right|=\left|y_{k} z_{k}\right| \text {. }
$$

But $y_{k}$ has a positive real value (recall that $y_{k}=\left(\gamma_{k} /\left(\gamma_{k}+\right.\right.$ $\left.\sigma^{2}\right)$ ), and $\sigma^{2}$ and $\gamma_{k}$ have positive real values). Thus, $\left|y_{k} z_{k}\right|=$ $\left|y_{k}\right|\left|z_{k}\right|=y_{k}\left|z_{k}\right|$. But, $z_{k}=\exp \left\{j(2 \pi / \lambda) d_{d} \times \cos \left(\theta_{k}\right)\right\}$, so it is clear that $\left|z_{k}\right|=1$. So, the magnitude of the eigenvalue $\left(\phi_{k}\right)$ is given by

$$
\left|\phi_{k}\right|=y_{k}=\frac{\gamma_{k}}{\gamma_{k}+\sigma^{2}}
$$

The noise variance effect on the $\phi_{k}$ can be measured by defining the variable $\rho_{k}$ which is defined as the absolute difference between $\left|\phi_{k}\right|$ and its noise-free version $\left(\left|\bar{\phi}_{k}\right|\right)$, where $\bar{\phi}_{k}=\left.\phi_{k}\right|_{\sigma^{2}=0}=1$. Thus, $\rho_{k}$ is defined as follows:

$$
\rho_{k}=|| \phi_{k}|-| \bar{\phi}_{k}||=\frac{\sigma^{2}}{\gamma_{k}+\sigma^{2}} .
$$

Clearly, the minimum value for $\rho_{k}$ is 0 when $\sigma^{2}=0$. Whereas its maximum value is 1 as $\sigma^{2} \rightarrow \infty$.

As for the TLS-ESPRIT algorithm, the AOA estimation starts by taking the EVD of an autocorrelation matrix of the received signals (call it $\widetilde{\mathbf{R}}_{\mathrm{TLS}}$ ) [9]. Let $\mathbf{R}_{\mathrm{TLS}}$ be defined as the noise-free version of $\widetilde{\mathbf{R}}_{\mathrm{TLS}}$. Also, let $\bar{\mu}_{k}$ for $k=1 \rightarrow K$ be one of the matrix $\mathbf{R}_{\mathrm{TLS}}$ eigenvalues. Then, each eigenvalue of $\widetilde{\mathbf{R}}_{\mathrm{TLS}}$ (which is given the notation $\mu_{k}$ ) will be given as follows:

$$
\mu_{k}=\bar{\mu}_{k}+\sigma^{2} \text {. }
$$

Defining $\epsilon_{k}$ as the absolute difference between $\left|\mu_{k}\right|$ and its noise free version $\left(\left|\bar{\mu}_{k}\right|\right)$, then,

$$
\epsilon_{k}=\sigma^{2}
$$

Thus the minimum value for $\epsilon_{k}$ is 0 when $\sigma^{2}=0$. Whereas its maximum value approaches $\infty$ as $\sigma^{2} \rightarrow \infty$.

Comparing $\rho_{k}$ and $\epsilon_{k}$ in (46) and (48), respectively, it is clear that in low noise variance cases, the deviation between the estimated eigenvalues and the true eigenvalues will be small for both algorithms (CDBA and TLS-ESPRIT); thus, it is expected that both algorithms will have close performance in low noise variance cases (i.e., high SNR).

In high noise variance cases, the deviation between the estimated eigenvalues and the true eigenvalues will have a maximum value of 1 in CDBA algorithm, whereas, in the TLS-ESPRIT the deviation will approach $\infty$. Recall that the CDBA algorithm estimates the AOAs from the eigenvalues $\left(\phi_{k}\right)$ for $k=1 \rightarrow K$, and the TLS-ESPRIT estimates 


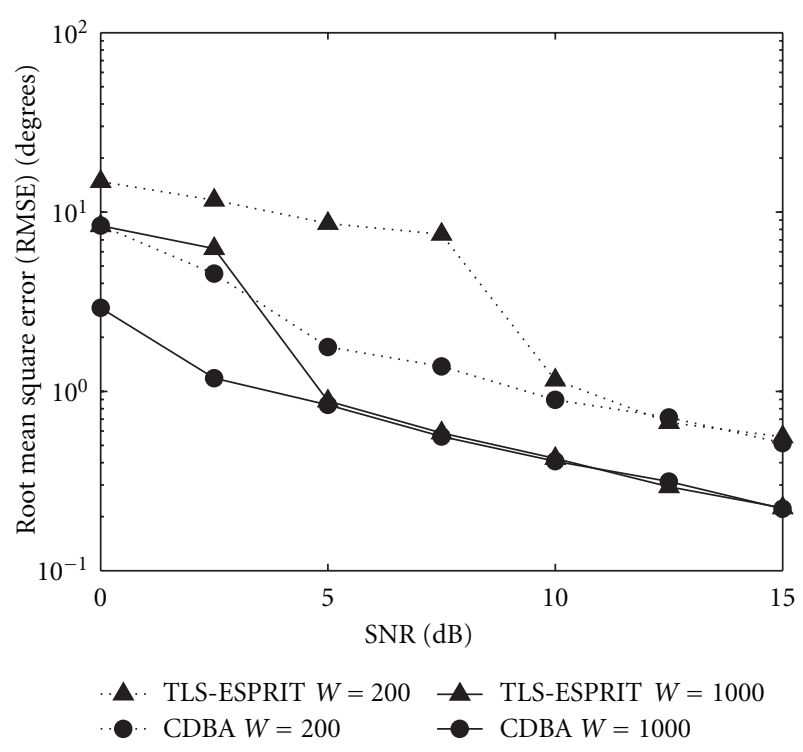

FIgURe 1: Root mean square error (RMSE) of angle estimation in degrees versus signal-to-noise-ratio (SNR) in $\mathrm{dB}$ for $\left(\theta_{1,2}=\right.$ $\left.30^{\circ}, 75^{\circ}\right)$.

the AOAs from the eigenvectors of $\widetilde{\mathbf{R}}_{\mathrm{TLS}}$ (see [9] for more insight), and any error in estimating the eigenvalues of $\widetilde{\mathbf{R}}_{\mathrm{TLS}}$ will directly change their corresponding eigenvectors causing an error in estimating the AOAs. Also, it is clear that in high noise variance cases (i.e., low SNR) the EVD process in the CDBA algorithm is much less affected by the noise than the EVD process in the TLS-ESPRIT algorithm. Thus, it is expected that the CDBA algorithm will perform better than the TLS-ESPRIT in high noise variance cases (i.e., low SNR).

The performance of the proposed CDBA method will be shown in the simulation section (Section 4).

\section{Simulation Results}

Simulations for the proposed CDBA estimator were completed to assess its performance. The results in this section were averaged over 1000 ensemble runs. The elements of each antenna array were separated by a half-wavelength (i.e., $d=$ $\lambda / 2$ ), and without any loss of generality the distance between the elements of each antenna doublet $\left(d_{d}\right)$ was chosen to be $\lambda / 2$ as well. The number of sources was set to $2 . A_{1}$ was set to 1.2 , and $A_{2}$ was set to 1 . The proposed CDBA method was compared with the TLS-ESPRIT method of [9].

Figure 1 shows the root-mean-square error (RMSE) of the AOA estimation in degrees for the proposed CDBA method compared with the TLS-ESPRIT method for different SNRs. Since the power for both users was not equal, then the SNR was taken for the second user; that is, if the SNR was set to $5 \mathrm{~dB}$, then the second user would have a $5 \mathrm{~dB}$ SNR and the first user would have SNR of $20 \log 1.2+5=6.58$ $\mathrm{dB}$. The angles for the two users were set to $\theta_{1}=30^{\circ}$ and $\theta_{2}=75^{\circ}$. The number of antenna doublets was set to $M=3$. The number of snap shots was set to $W=200$ and $W=1000$ (where $W$ is the number of snap shots over which

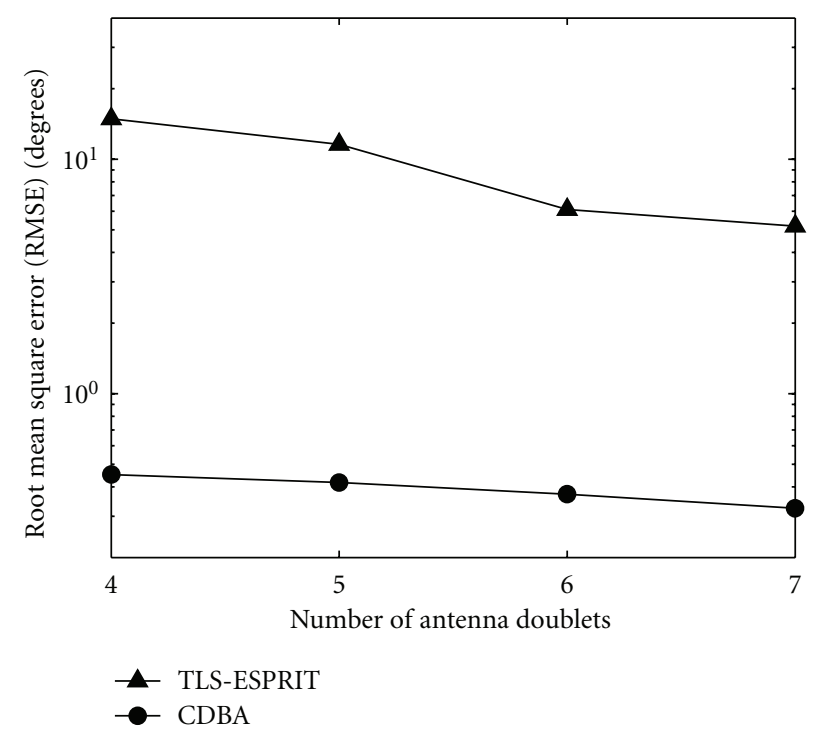

FIGURE 2: Root mean square error (RMSE) of angle estimation in degrees versus number of antenna doublets for $\left(\theta_{1,2}=30^{\circ}, 75^{\circ}\right)$.

TABLE 1: Root mean square error (RMSE) of angle estimation in degrees for different AOA separations.

\begin{tabular}{lcc}
\hline AOA separation $(\delta)$ & TLS-ESPRIT & CDBA \\
\hline $1^{\circ}$ & $11.5223^{\circ}$ & $3.1770^{\circ}$ \\
$1.5^{\circ}$ & $6.8057^{\circ}$ & $1.6336^{\circ}$ \\
$2^{\circ}$ & $4.2739^{\circ}$ & $0.7330^{\circ}$ \\
\hline
\end{tabular}

the correlation matrices were estimated). The results in Figure 1 show that the proposed CDBA method gave better performance than the TLS-ESPRIT method in low SNR cases. This result agrees with the discussion presented in Section 3.2.

Figure 2 shows the RMSE of the AOA estimation in degrees for the proposed CDBA method compared with the TLS-ESPRIT method for different number of antenna doublets. The angles for the two users were set to $\theta_{1}=$ $30^{\circ}$ and $\theta_{2}=75^{\circ}$. The number of snap shots was set to $W=200$. The results in Figure 2 show that the CDBA method outperformed the TLS-ESPRIT algorithm for different number of antenna doublets.

Another important issue in comparing AOA estimators is their performance when the AOAs of the received signals are close to each other. Table 1 shows the RMSE of the AOA estimation in degrees for the proposed CDBA method compared with the TLS-ESPRIT method for different AOA separations for two users. The number of antenna doublets was set to $M=6$. The number of snap shots was set to $W=200$. The angular deviation was performed by setting $\theta_{1}$ to be taken from the following equation: $\theta_{1}=\theta_{2}+\delta$ where $\delta$ is the angular deviation with $\theta_{2}=75^{\circ}$. Table 1 shows that the CDBA method outperformed the TLS-ESPRIT algorithm for different AOA separations.

Also, to compare both methods computational complexity, two functions in MATLAB (tic.m and toc.m) were 
used to measure the time it takes each method to estimate the AOA. The CDBA and the TLS-ESPRIT methods took 0.281 and 0.297 seconds, respectively, to perform one run of AOA estimation for two users. Clearly, this indicates that the CDBA method has less computational complexity than the TLS-ESPRIT method.

\section{Conclusion}

In this paper, an AOA estimator is proposed which is named the CDBA method. The CDBA method is applied by taking the Cholesky decomposition of the received signal autocorrelation matrix. The resultant decomposed matrices are used to modify the crosscorrelation matrix of the received signals at the antenna array doublets. The proposed CDBA method has better performance than the TLS-ESPRIT method in estimating the AOAs especially in low SNR cases. The performance of the proposed CDBA method was assessed and compared to the TLS-ESPRIT method.

\section{References}

[1] N. Hew and N. Zein, "Space-time estimation techniques for UTRA system," in Proceedings of the IEE Colloquium on Capacity and Range Enhancement Techniques for the 3rd Generation Mobile Communications and Beyond (Ref. No. 2000/003), pp. 6/1-6/7, London, UK, 2000.

[2] Y. F. Chen and M. D. Zoltowski, "Joint angle and delay estimation for DS-CDMA with application to reduced dimension space-time rake receivers," in Proceedings of the IEEE International Conference on Acoustics, Speech, and Signal Processing (ICASSP '99), vol. 5, pp. 2933-2936, March 1999.

[3] S. Al-Jazzar, M. Ghogho, and D. McLernon, "A joint TOA/AOA constrained minimization method for locating wireless devices in non-line-of-sight environment," IEEE Transactions on Vehicular Technology, vol. 58, no. 1, pp. 468-472, 2009.

[4] P. Deng and P. Fan, "An AOA assisted TOA positioning system," in Proceedings of the 2000 International Conference on Communication Technology Proceedings (WCC-ICCT '00), vol. 2, pp. 1501-1504, Beijing, China, August 2000.

[5] Y. H. Choi, "Maximum likelihood estimation for angles of arrival of coherent signals using a coherency profile," IEEE Transactions on Signal Processing, vol. 48, no. 9, pp. 2679-2682, 2000.

[6] X. Huang and Y. J. Guo, "Frequency-domain AoA estimation and beamforming with wideband hybrid arrays," IEEE Transactions on Wireless Communications, vol. 10, no. 8, pp. 2543-2553, 2011.

[7] J. Verhaevert, E. V. Lil, and A. V. de Capelle, "Direction of arrival (DOA) parameter estimation with the SAGE algorithm," Signal Processing, vol. 84, no. 3, pp. 619-629, 2004.

[8] Q. Cheng, R. Yang, and H. Zhang, "Optimally weighted ESPRIT using uniform circular arrays," Computers and Electrical Engineering, vol. 31, no. 4-5, pp. 272-281, 2005.

[9] R. Roy and T. Kailath, "ESPRIT-estimation of signal parameters via rotational invariance techniques," IEEE Transactions on Acoustics, Speech, and Signal Processing, vol. 37, no. 7, pp. 984995, 1989. 

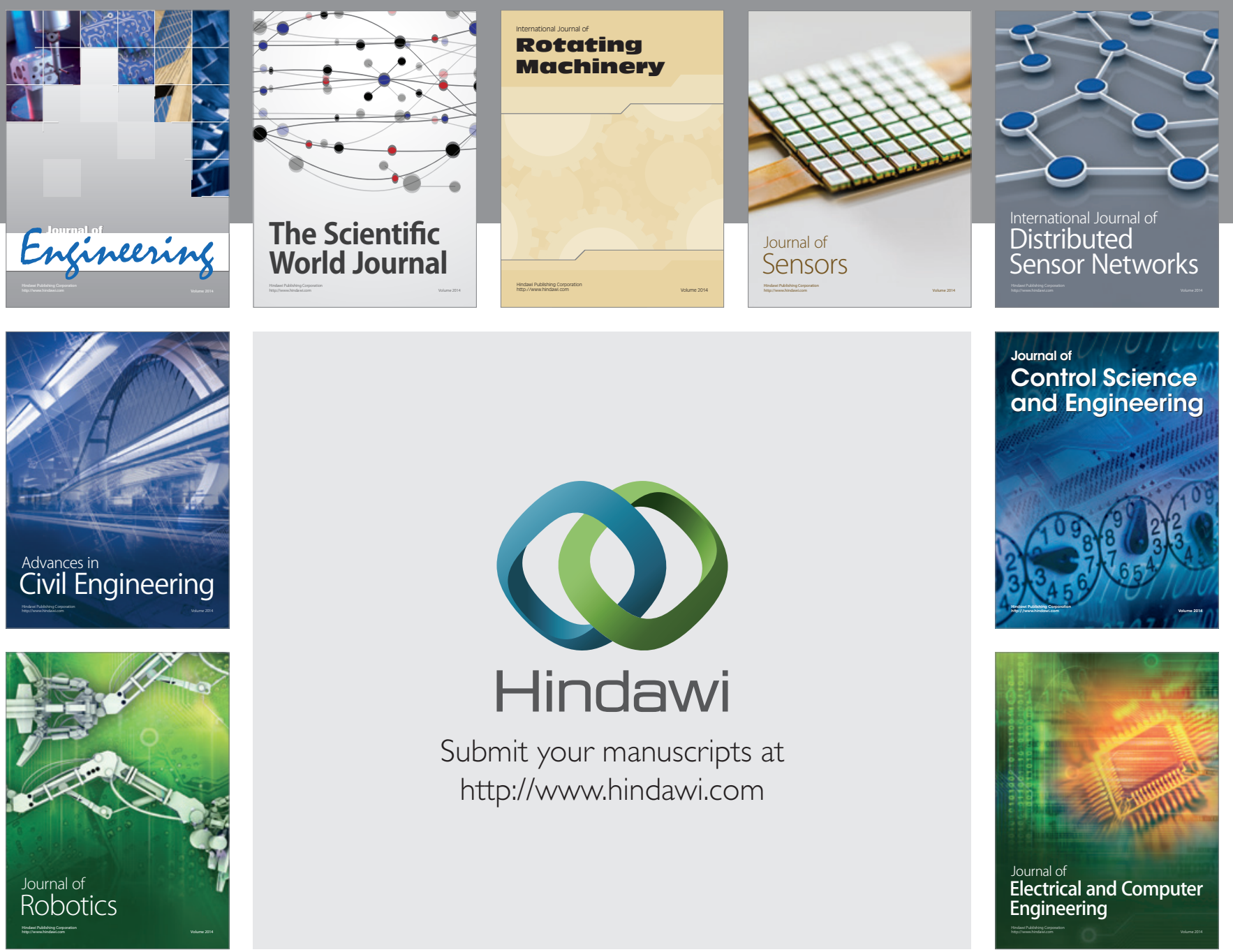

Submit your manuscripts at

http://www.hindawi.com
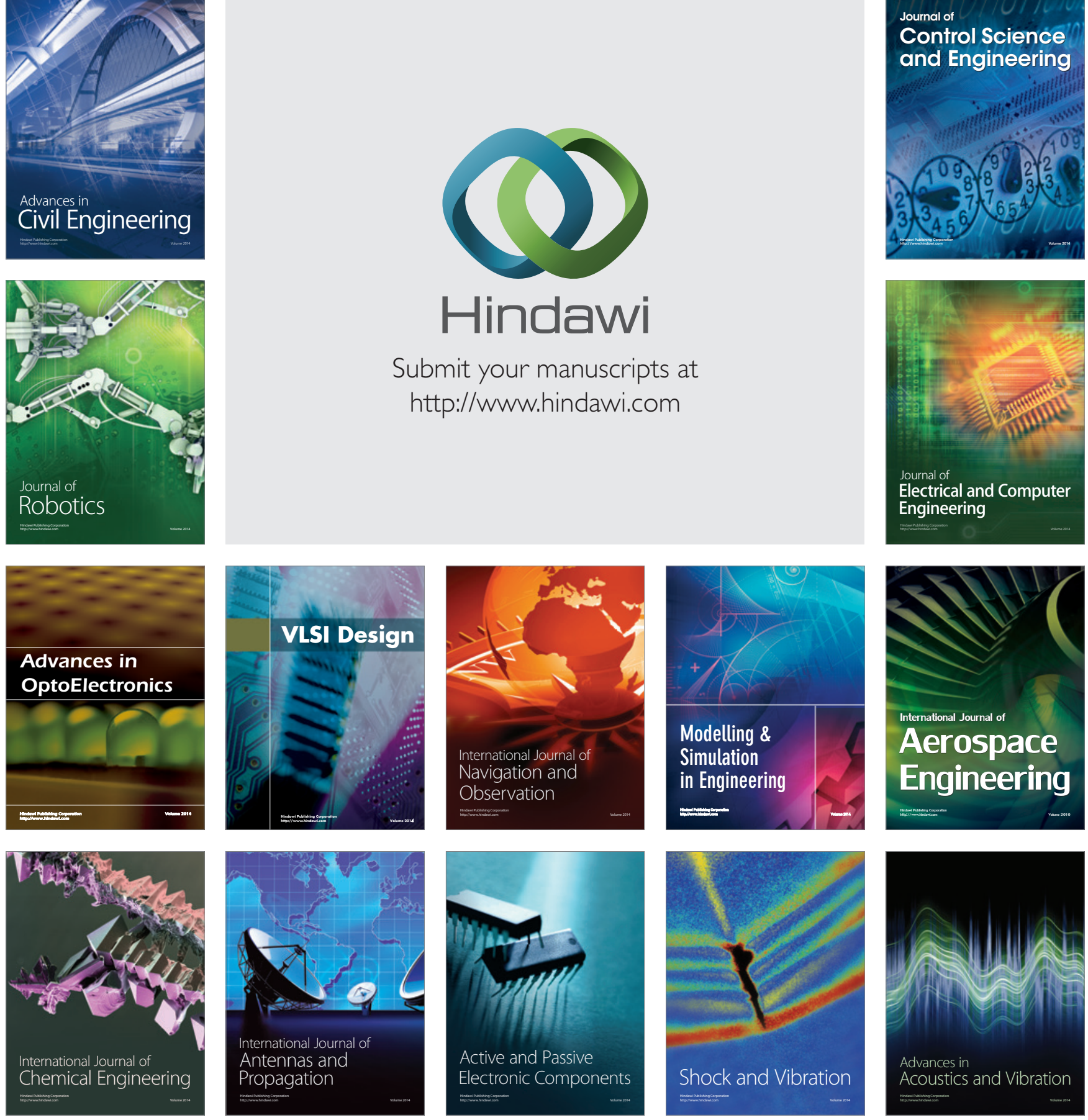